\title{
Mesenchymal stem cell carriers enhance anti-tumor efficacy of oncolytic virotherapy (Review)
}

\author{
XIANYAO WANG ${ }^{1-3}$, XING ZHAO ${ }^{1-3}$ and ZHIXU HE ${ }^{2,4}$ \\ ${ }^{1}$ Center for Tissue Engineering and Stem Cell Research, Guizhou Medical University; \\ ${ }^{2}$ Key Laboratory of Adult Stem Cell Translational Research, Chinese Academy of Medical Sciences, Guiyang, \\ Guizhou 550004; ${ }^{3}$ Department of Immunology, Guizhou Medical University, Guiyang, Guizhou 550025; \\ ${ }^{4}$ Department of Pediatrics, Affiliated Hospital of Zunyi Medical University, Zunyi, Guizhou 563000, P.R. China
}

Received July 19, 2020; Accepted December 9, 2020

DOI: $10.3892 / \mathrm{ol} .2021 .12499$

\begin{abstract}
Oncolytic viruses (OVs) specifically infect, replicate and eventually destroy tumor cells, with no concomitant toxicity to adjacent normal cells. Furthermore, OVs can regulate tumor microenvironments and stimulate anti-tumor immune responses. Mesenchymal stem cells (MSCs) have inherent tumor tropisms and immunosuppressive functions. MSCs carrying OVs not only protect viruses from clearing by the immune system, but they also deliver the virus to tumor lesions. Equally, cytokines released by MSCs enhance anti-tumor immune responses, suggesting that MSCs carrying OVs may be considered as a promising strategy in enhancing the anti-tumor efficacies of virotherapy. In the present review, preclinical and clinical studies were evaluated and discussed, as well as the effectiveness of MSCs carrying OVs for tumor treatment.
\end{abstract}

Correspondence to: Dr Zhixu He, Key Laboratory of Adult Stem Cell Translational Research, Chinese Academy of Medical Sciences, 9 Beijing Road, Guiyang, Guizhou 550004, P.R. China

E-mail: hzx@gmc.edu.cn

Dr Xing Zhao, Center for Tissue Engineering and Stem Cell Research, Guizhou Medical University, 9 Beijing Road, Guiyang, Guizhou 550004, P.R. China

E-mail: xingzhao@gmc.edu.cn

Abbreviations: MSCs, mesenchymal stem cells; OVs, oncolytic virus; OVT, oncolytic virotherapy; TME, tumor microenvironment; TAAs, tumor-associated antigens; TANs, tumor-associated neoantigens; DAMPs, danger-associated molecular patterns; PAMPs, Pathogen-associated molecular patterns; OAD, oncolytic adenovirus; NK, natural killer; DC, dendritic cell

Key words: oncolytic virus, mesenchymal stem cells, cellular carriers, tumor tropism, immunosuppressive function, oncolytic virotherapy

\section{Contents}

1. Introduction

2. Mechanisms of oncolytic virotherapy

3. Main hurdles limiting the efficacy of OV for virotherapy

4. MSC biology

5. MSCs loaded with OVs: The basics and the anti-tumor story

6. Conclusions and perspectives

\section{Introduction}

With the development of targeted therapies and cellular immunotherapies, such as T cell-based, natural killer (NK) cell-based and dendritic cell (DC)-based immunotherapies, the therapeutic efficacy of cancer treatment has been greatly improved (1). However, the overall remission and survival rate of patients with certain tumors has not been fundamentally addressed. In recent decades, oncolytic viruses (OVs) have generated widespread interest, and have become a major focus of interest for clinicians and scientists $(2,3)$. These viruses include adenovirus, measles virus, reovirus, herpes simplex virus, Newcastle disease virus, vesicular stomatitis, vaccinia virus and poliovirus $(4,5)$.

Previous preclinical and clinical studies have demonstrated that the intratumoral injection of OVs is effective, although the efficacy toward disseminated and metastatic tumors remains modest $(6,7)$. Numerous factors can affect viral efficiency in reaching tumor tissue, including viral destruction by the immune system and viral absorption by tissues and organs $(8,9)$. Therefore, appropriate carrier vehicles are required to deliver OVs to tumor sites in order to improve therapeutic efficacy.

In recent years, mesenchymal stem cells (MSCs) have become a promising cellular vehicle for anti-tumor drug delivery, thanks to their inherent tumor tropism (10-13). MSCs can specifically migrate to the tumor or inflammatory site. A recent review has reported that MSCs can be modified by advanced approaches to suppress tumor growth (14). Furthermore, MSCs exert immunosuppressive functions, by inhibiting NK proliferation, cytotoxicity and cytokine production (15), suppressing differentiation and function of DC (16) and inducing therefore the emergence of regulatory 
T cells. These features make MSCs ideal candidates for OVs delivery. In the present review, an overview of MSC loading of OVs for oncolytic virotherapy was provided. We briefly introduced MSC characteristics for OV delivery and summarized developments in the MSC oncolytic virotherapy arena.

\section{Mechanisms of oncolytic virotherapy}

In the last decades, great progress has been made in elucidating the molecular mechanisms of OV infection. OVs can infect target cells using low-affinity binding to sialic acid residues, from where they internalize via specific high-affinity receptors $(17,18)$. The expression of OV strain receptors on the cell surface is a crucial factor in determining viral infection (19). However, accumulating evidence from preclinical and clinical studies has indicated that growth conditions and genetic background of tumor cells can affect cell sensitivity to OVs (19). For example, cathepsin B and cathepsin L are critical for viral shelling, which is associated with the sensitivity of tumor cells to oncolytic reoviruses; however, virus shelling is also limited by low levels of cathepsin B and cathepsin L in normal cells (20). In addition, Ras mutations can increase cell sensitization to reoviruses $(21,22)$. Following $\mathrm{OV}$ infection, virus progeny replicates highly in tumor cells, eventually lysing and killing infected cells. Subsequently, tumor cell lysis releases infectious viral progeny that spreads to surrounding tumor cells, causing more tumor cells to undergo oncolysis. However, OV replication is often limited in healthy cells, thus viral clearance is rapid with minimal oncolysis (23).

With expanding OV research, virotherapy has gradually changed from direct oncolysis to virus mediated anti-tumor immunity $(24,25)$. It has been demonstrated that the immune system serves a crucial role in oncolytic virotherapy. On the one hand, inherent and adaptive immunities control viral infections, reducing or eliminating their oncolytic potential. On the other hand, viruses can trigger anti-tumor immune responses through a variety of mechanisms. Firstly, tumor-associated antigens (TAAs) and neoantigens (TANs), which are released by tumor cells, are captured by antigen-presenting cells and are ultimately activated by tumor specific $\mathrm{T}$ cells in order to respond to tumor antigens $(26,27)$. Secondly, OVs can promote immunogenic cell death by cell necrosis, immunogenic apoptosis and autophagic cell death (27-30), subsequently releasing danger-associated molecular patterns (DAMPs), including ATP and high-mobility group box 1 protein $(28,31,32)$. In addition, virus-induced tumor cell death also leads to the release of pathogen-associated molecular patterns (PAMPs), such as nucleic acids, proteins and viral capsid components $(33,34)$. DAMPs and PAMPs are recognized by pattern recognition receptors (PRRs) on innate immune cells, such as DC and NK cells, in turn activating NF- $\kappa \mathrm{B}$ signaling and releasing type I interferon (IFN), proinflammatory cytokines and chemokines $(35,36)$. However, these molecules promote the recruitment and activation of macrophages, NK, DC and tumor specific cytotoxic T lymphocytes to the tumor microenvironment (TME), and help reverse the immunosuppressive state of TME (32,35-38). In addition, tumor cells infected with OVs express virus-specific antigens on their surface, which facilitate their destruction by anti-viral T cells (39). Therefore,
OVs can induce anti-tumor immune response, even if the virus does not effectively replicate (40).

\section{The main hurdles limiting OV efficacy for virotherapy}

In 2015, the US Food and Drug Administration approved Amgen's talimogene laherparepvec (T-VEC or Imlygic ${ }^{\circledR}$ ) for the treatment of melanoma (41), and in December of the same year, T-VEC was approved by the European Medicines Agency for the treatment of unresectable stage IIIB/C and stage IVM1a melanoma (42). The T-VEC success has significantly promoted $\mathrm{OV}$ research and clinical applications, and aroused great interest in the academic and industry communities $(43,44)$. However, in most cases, the elicited immune response limits the killing effects of OVs, the efficacy remains modest, and the ultimate therapeutic efficacy of OVs as a systemic administration reagent is limited (45-47). There are four reasons that may explain this phenomenon: i) Individuals carry anti-viral antibodies, such as anti-reovirus and anti-measles virus antibodies. After systemic administration, OVs are quickly cleared by pre-existing antibodies, which hinders OV efficacy $(48,49)$; ii) OVs are cleared by macrophages located in the liver and spleen; iii) for solid tumors, OVs must pass through the endothelial layer to reach target cells, therefore physical barriers pose a significant challenges to viral transmission; and iv) due to interactions between OVs and antigen presenting cells, extensive anti-viral immunity, pre-existing circulating antibodies and blood factors, such as coagulation factors and complement proteins, OVs are easily cleared by the host's immune system (50). Taken together, these factors suggest that it may be difficult to determine whether enough OV particles could reach the tumor site. In the following sections of this review, current strategies for OVs loading by MSCs for anti-tumor therapy will be discussed.

\section{MSC biology}

MSCs are adult stem cells derived from the mesoderm that can be isolated from various tissues, including bone marrow, adipose tissue, dental pulp, placenta, amniotic fluid, umbilical cord, Wharton's jelly and umbilical cord blood (51,52) (Fig. 1). Although MSCs derived from these tissues contain diverse background genetic lineages, they can exert intrinsic and extrinsic effects, and MSCs cultured in vitro may share common features in agreement with the International Society of Cell Therapy (ISCT) criteria established in 2006 (53). Firstly, under in vitro culture conditions, MSCs exhibit spindle-shaped or fusiform morphology. Secondly, in vitro cultured MSCs express CD73, CD90 and CD105 markers on their surface; however, they express no monocyte markers, such as HLA-DR, CD14 or CD11b, CD79 $\alpha$ or CD19, and no hematopoietic markers, such as CD34 and CD45 (53). In addition, MSCs can differentiate into osteoblasts, adipocytes and chondroblasts following specific in vitro differentiation conditions (53). Although MSCs have the potential to express surface antigens and differentiate, other characteristics of MSCs that would support anti-tumor therapeutic interests are vital. In the following section, MSC functions, including inherent tumor tropisms, as well as the immunosuppression and paracrine characteristics of anti-tumor MSC carrying OVs will therefore be discussed. 


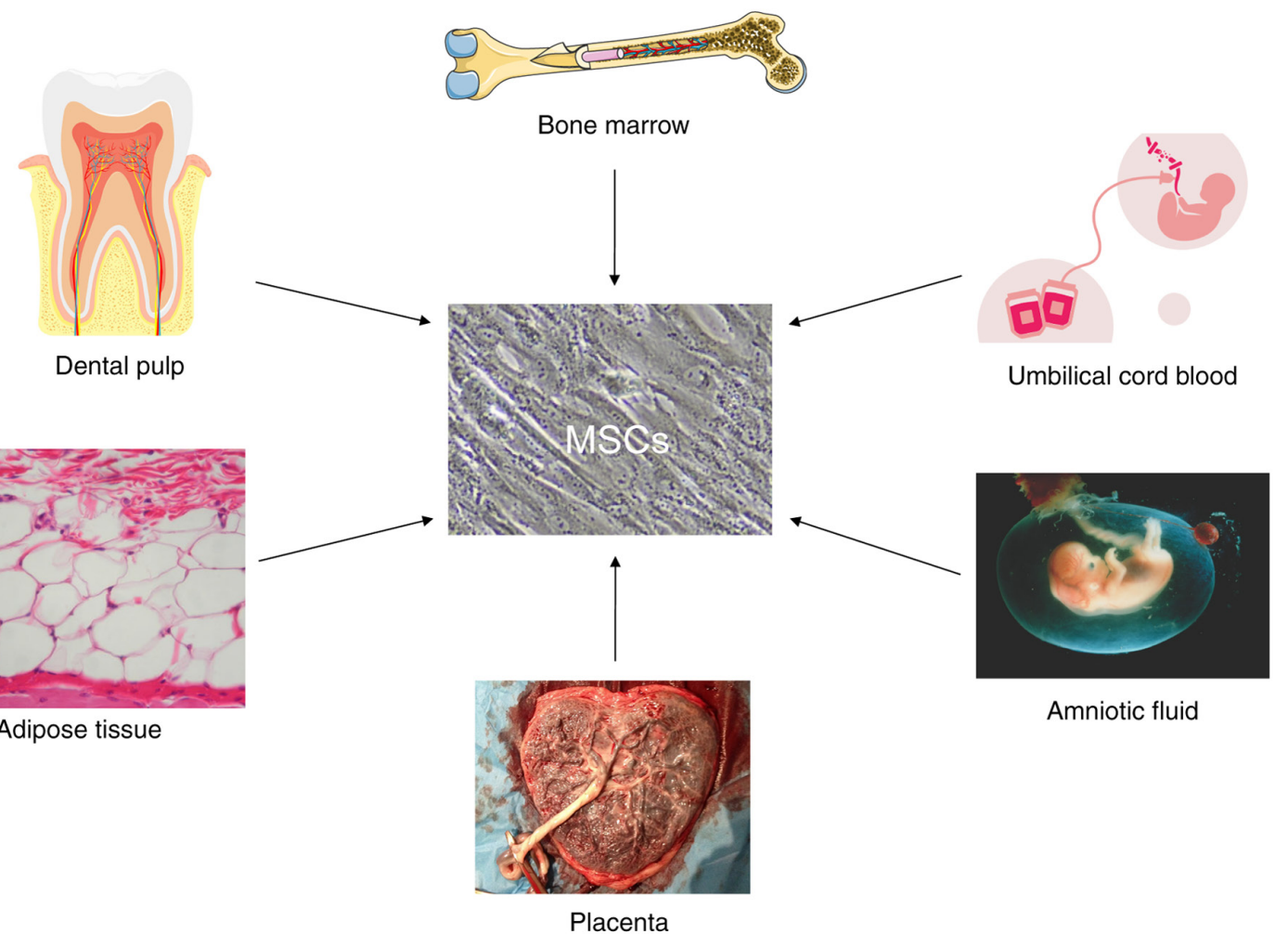

Figure 1. Different sources of MSCs in humans. MSCs, mesenchymal stem cells.

\section{MSCs loaded with OVs-the anti-tumor story}

MSC tumor tropisms facilitate $\mathrm{OV}$ delivery to tumor sites. MSCs undergo chemotaxis and migration to tumor lesions (54). A recent study has reported that MSCs migrate and bind to the tumor matrix and target the TME (14). At these sites, the tumor oxidation state, vascularization and tumor inflammatory status can affect MSC migration efficiency (55). Furthermore, MSCs have been demonstrated to exert positive chemotactic effects on solid tumors, such as hepatocellular carcinoma (55), breast cancer (56) and glioma (57).

MSCs migrate to damaged tissue or inflammatory sites and release simultaneous secretory cytokines $(58,59)$. In addition to tumor cells, the TME also contains immune cells, fibroblasts, vascular endothelial cells, adipocytes and tumor stromal cells, which secrete large numbers of cytokines, such as vascular endothelial growth factor (VEGF), platelet derived growth factor (PDGF), interleukin (IL)-8, IL-6, stromal cell-derived factor-1 (SDF-1), basic fibroblast growth factor (bFGF), granulocyte colony-stimulating factor (G-CSF), granulocyte-macrophage colony-stimulating factor (GM-CSF), monocyte chemoattractant protein-1 (MCP-1), hepatocyte growth factor (HGF), tumor necrosis factor- $\alpha$ (TNF- $\alpha$ ), transforming growth factor- $\beta$ (TGF- $\beta$ ), urokinase type plasminogen activator receptor, vascular cell and intercellular cell adhesion molecules (VCAM, ICAM), C-X-C motif chemokine ligand-12 (CXCL-12), C-C motif chemokine ligand-2 (CCL-2), C-C motif chemokine ligand-3 (CCL-3), $\mathrm{C}-\mathrm{C}$ motif chemokine receptor 4 (CCR4) and $\mathrm{C}-\mathrm{X}-\mathrm{C}$ motif chemokine receptor 4 (CXCR4) (59-63).

Pavon et al (64) reported that human umbilical cord blood-derived MSCs express the chemokine receptors CCR2 and CXCR4, and demonstrated that MCP-1/CCL2 and SDF-1/CXC12 secreted by CD133-positive GBM cells can induce MSC migration in vitro. Furthermore, in vivo experiments confirmed that MSCs can cross the blood-brain barrier and migrate to glioblastoma tumor areas (64). In addition, Lejmi et al (63) co-cultured hepatoma cells with MSCs and demonstrated that the expression of matrix metalloproteinase-1 is significantly increased in MSCs, promoting therefore MSCs migration toward hepatoma cells. In essence, cytokines secreted by immune and tumor cells are key to inducing the chemotactic migration of MSCs and are the central theoretical tenet for MSCs as OV cellular vehicles $(65,66)$. Therefore, when OVs are loaded onto MSCs, they exploit the inherent tumor tendency of MSCs to reach tumor sites, thereby increasing OV targeting and enhancing oncolysis.

MSC immunosuppressive functions protect $\mathrm{OV}$ clearance from the immune system. MSC immunological characteristics serve crucial roles in the therapeutic efficacy of MSCs loaded with OVs towards tumors. Evidence indicates that MSCs amplified in vitro do not express HLA-II or costimulatory molecules, such as CD40, CD80, CD83, CD86 and CD154 (67). Therefore, no additional immunosuppressants are required for autologous or allogeneic MSC transplantation. In addition, MSCs exert strong immunosuppressive functions. For example, MSCs produce and release a variety of soluble cytokines, including IL-6, IL-10, TGF- $\beta$, heme oxygenase-1, inducible nitric oxide synthase and indoleamine-2-dioxygenase-3 (68), which play major roles in immunosuppression. At present, MSCs are used for immunomodulation, mostly for immune rejection and autoimmune diseases, such as hematopoietic stem cell transplantation, organ transplantation, rheumatoid arthritis 
and systemic lupus erythematosus $(69,70)$. However, the underlying mechanisms of MSC immunosuppressive function in vivo remain unclear.

In recent years, increasing evidence from preclinical and clinical studies has indicated that MSCs exert immunosuppressive functions by inhibiting the activity of certain types of immune cell, including T, B lymphocytes and NKs, thereby affecting monocytes, DC and macrophage function (71-74). MSCs affect the activation, proliferation, maturation, cytokine production and cytotoxic activity of innate and adaptive immune cells (68). Indeed, MSCs can reduce cytokine secretion from helper $\mathrm{T}$ cells, weaken the killing effects of effector $\mathrm{T}$ lymphocytes (75), hinder B lymphocyte differentiation and impede their ability to secrete immunoglobulin $(76,77)$, and inhibit INF- $\gamma$ secretion by NK cells and reduce their killing effects (78). In addition, MSCs prevent $\mathrm{CD} 14^{+}$monocytes and $\mathrm{CD}_{3} 4^{+}$progenitor cells from differentiating into mature DC cells (79). Importantly, MSCs promote the emergence of regulatory immune subsets, including $\mathrm{CD} 8^{+} \mathrm{CD} 28^{-} \mathrm{T}$ lymphocytes $(80), \mathrm{CD}^{+} \mathrm{CD} 25^{+} \mathrm{FOXP} 3^{+} \mathrm{T}$ lymphocytes (81), IL-10-producing B lymphocytes (82) and IL-10-producing DCs (83). Therefore, inhibiting immune cell functions and promoting the emergence of regulatory immune cell subsets, could serve positive roles in MSC immunosuppressive functions. These functions are key MSC features in protecting OVs from immune system clearance, and a guarantee to enhance OV spread and increase viral persistence (84).

MSC carriers induce systemic anti-tumor immune responses. It has been reported that MSCs promote tumorigenesis through various mechanisms, such as inhibition of local immune responses (51), stimulation of epithelial-mesenchymal transformation, inhibition of tumor cell apoptosis and promotion of angiogenesis and tumor metastasis (85). Previous studies have demonstrated that MSCs, in contrast to their tumorigenic functions, can inhibit tumor growth by inhibiting angiogenesis (86), inducing cell cycle arrest $(14,87)$, enhancing inflammatory infiltration (88) and inhibiting proliferation-associated signaling pathways (14).

Although there is some controversy over whether MSCs inhibit or promote tumor growth, emerging evidence indicates that oncolytic adenovirus (OAD)-infected MSCs induce anti-tumor immune responses and increase leukocyte infiltration into tumor lesions (89). Similarly, Mahasa et al (10) predicted the therapeutic efficacy of MSCs loaded with OAD in a Hep3B cell tumor model using an integrated mathematical-experimental model, and demonstrated that MSCs loaded with OAD can promote tumor therapeutic efficacy. In addition, a phase I clinical trial (NCT01844661) of bone marrow-derived MSCs carrying Celyvir for the treatment of metastatic or refractory tumors was completed and reported that the combination of MSCs and Celyvir is safe (90). Following treatment with MSCs carrying Celyvir, except for the increase in the amount of oncolytic virus administered to patients, minimizing toxicities and avoiding direct tumor injections, no grades 2-5 toxicities were reported (90). However, the safety and efficacy of MSCs carrying Celyvir require further evaluation in a phase II setting.

Mechanically, after MSC infection with the human OAD icovir-5 in vitro, the NF- $\mathrm{KB}$ signaling pathway is activated and releases large numbers of cytokines, such as IL-6, CXCL2, CXCL10 and CCL5 (91). These cytokines facilitate the migration of $\mathrm{NK}$ and $\mathrm{T}$ cells, amongst others, to the TME (Fig. 2). Indeed, $48 \mathrm{~h}$ following Celyvir transplantation, the levels of peripheral blood monocytes, NK cells and neutrophils are increased (89). Furthermore, the first-in-child trial of autologous MSCs infected with the human OAD icovir-5 (Celyvir) demonstrated that the number of circulating B-lymphocytes and dendritic cells is significantly higher in pediatric patients, and that CD4 and CD8 T lymphocytes are also higher in children at most time points, compared with adult cohorts (90). These preclinical data illustrate that MSCs can release cytokines that might promote anti-tumor immune responses mediated by OVs. These data are instrumental in encouraging more virotherapy preclinical and clinical studies, investigating the utility of MSCs as OV carriers for patients with advanced cancer.

MSCs as carriersfordelivering OVs. The majority of preclinical studies indicate efficacy factors for MSCs as carriers for OV delivery (92-94). Du et al (95) used MSCs as cellular carriers for oncolytic herpes simplex virus (HSV) in order to assess efficacy in immune-deficient and immune-competent mouse melanoma metastasis models. The results demonstrated that transplanted MSCs carrying HSV could migrate to the tumor site and significantly prolong mouse survival. Furthermore, in immune-competent mice, the combination of MSC-HSV and the anti-programmed death ligand 1 (anti-PD-L1) immune checkpoint inhibitor could increase $\mathrm{CD} 8^{+} \mathrm{T}$ lymphocyte infiltration, leading to the production of IFN- $\gamma$ and significant prolongation of mouse survival.

For enveloped OVs, MSCs can deliver viruses to tumor sites via hetero-cellular fusion. Ong et al (96) loaded bone marrow-derived MSCs with oncolytic measles virus, and co-cultured them with human hepatocellular carcinoma cells in vitro. The results demonstrated that syncytia number increases when MSCs carries the measles virus, which is not the case with non-enveloped virus. Furthermore, in the presence of high titer anti-measles virus antibodies, virus-infected MSCs significantly induce heterocellular formation when compared with naked virus. In addition, MSCs precisely deliver the measles virus to tumor lesions in a patient-derived hepatocellular carcinoma model (96). These results were consistent with Castleton et al (97) who reported MSC delivery of the measles virus in a model for acute lymphoblastic leukemia, suggesting that OV infected MSCs could significantly prolong survival and improve anti-tumor efficacy when compared with the naked virus.

In addition, genetic engineering improves MSC delivery efficiency, enhances viral oncolytic activity and reduces virotherapy side effects. Yoon et al (55) reported that the OAD infection capability of MSCs is enhanced after modification of the fiber domain of OADs, allowing the virus to replicate efficiently in MSCs. These MSCs infected with OADs could effectively lyse hepatocellular carcinoma cells in vitro. Importantly, following MSC-OAD transplantation, MSCs home to the tumor site, facilitating a high accumulation of virions at the site, and ultimately leading to tumor growth inhibition. In another study, Kaczorowski et al (66) deleted the anti-apoptotic gene E1B19K from OAD and inserted the 

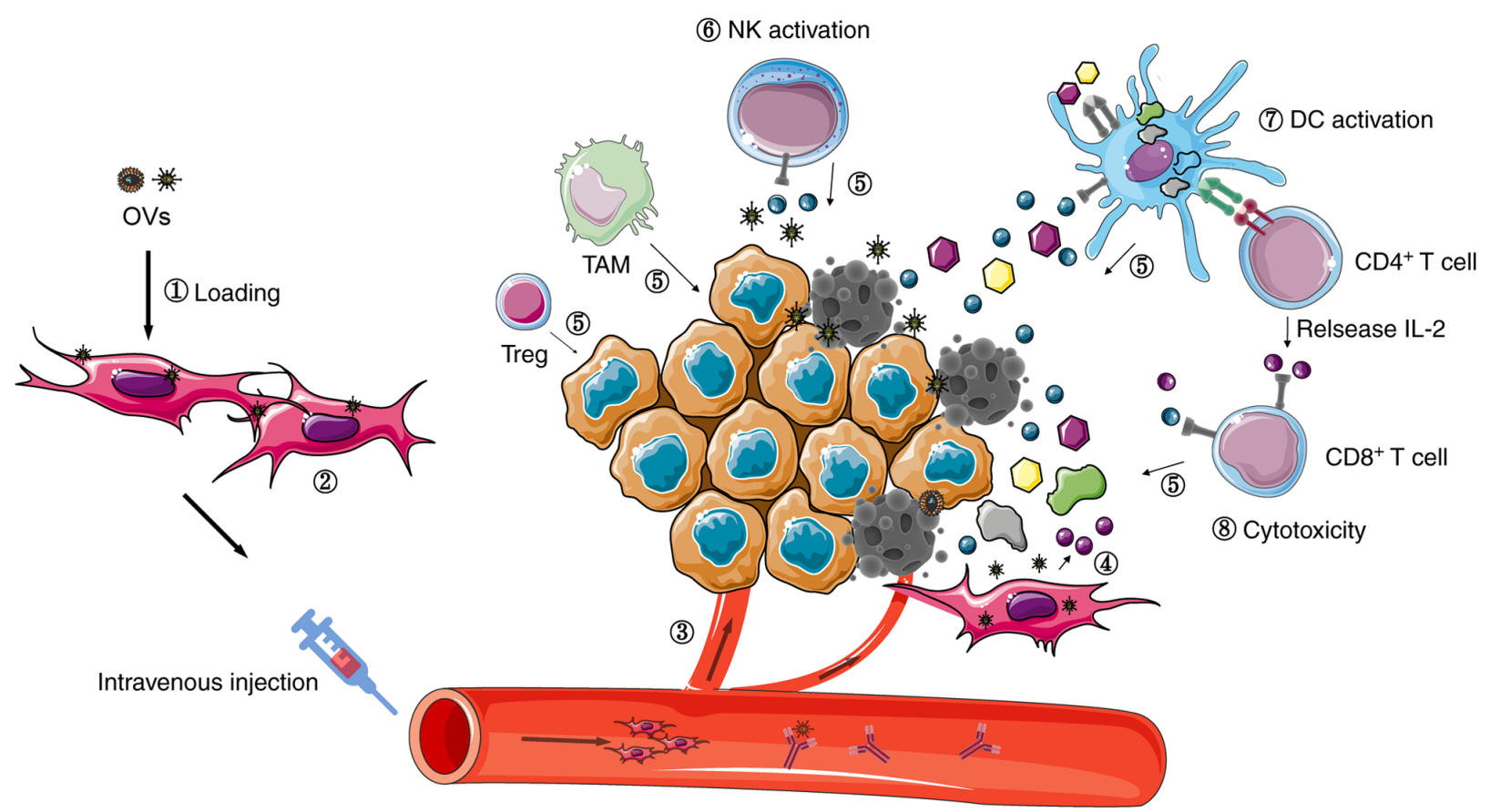

(8) Cytotoxicity

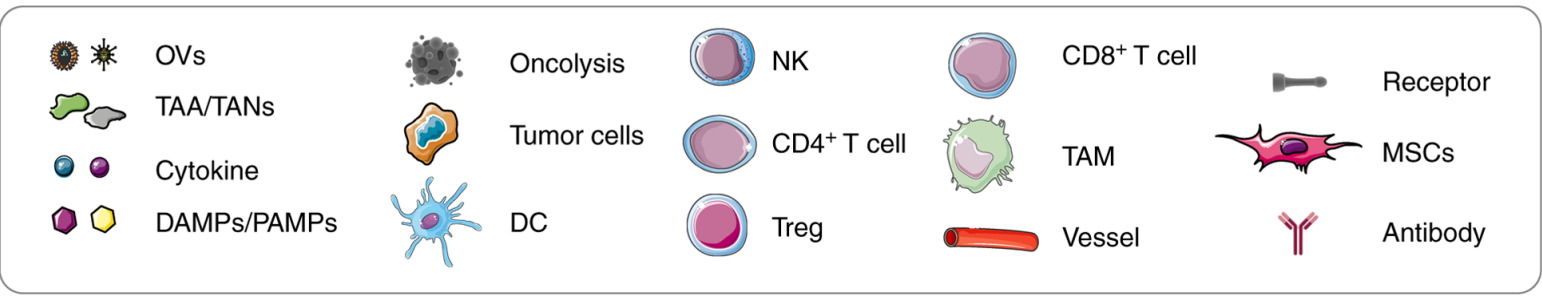

Figure 2. MSC carriers enhance anti-tumor efficacy of oncolytic virotherapy. (1) MSCs loaded with OVs. (2) MSCs provide a replication locale for OVs to produce more virus particles. (3) Tumor tropisms and immunosuppressive MSC functions facilitate precise OV targeting to tumor lesions. OVs infect tumor cells and release 'dangerous' signals. (4) OVs alter MSC cytokine profiles. (5) Cytokines induce immune cell migration to the TME. (6) NK activation. (7) DCs activation. (8) Tumor antigen specific T cell activation. OVs, oncolytic virus; DC, dendritic cell; MSCs, mesenchymal stem cells; DAMPs, danger-associated molecular patterns; PAMPs, Pathogen-associated molecular patterns; TAAs, tumor-associated antigens; TANs, tumor-associated neoantigens; NK, natural killer; TAM, tumor-associated macrophage; TME, tumor microenvironment.

cell death ligand TRAIL gene of OAD. After intravenous injection of virally infected MSCs, adenovirus capsid protein is detected in tumor xenografts established by cancer stem cell of pancreatic ductal adenocarcinoma. Similarly, following viral MSC treatment, the tumor size decreases significantly, the tumor cell proliferation-associated Ki67 and CD24 expression decreases and the tumor cell apoptosis-associated caspase- 3 activity increases (66). In addition, OADs significantly increase virus release from MSCs following the deletion of the anti-apoptotic virus gene E1B19K, or the overexpression of the cell death ligand TRAIL, while MSC migration ability remains unaffected (98). These data suggest that genetic modification of OADs can induce effective oncolysis, which may represent a promising strategy for OVs in clinical applications. Similarly, MSCs as carriers for the delivery of genetically modified OVs may be considered as a useful method for improving oncolytic virotherapy efficacy (Table I)(99-106). However, MSCs can also be modified by genetic modification or preconditioned to modification in order to improve their inherent properties, such as enhanced migration, adhesion and survival, and reduced premature senescence (107). OV delivery and virotherapy efficacy may therefore be improved.

\section{Conclusions and perspectives}

In summary, MSCs enhance the anti-tumor efficacy of virotherapy through numerous factors. Firstly, MSCs provide a replication location for OVs, facilitating the production of more virus particles, which is beneficial for virotherapy. Secondly, the tumor tropism and immunosuppression function of MSCs allow the virus to accurately reach the tumor site and enhance the transmission and persistence of the virus. Thirdly, oncolysis leads to the release of 'dangerous' signals, such as TAAs/TANs and DAMPs/PAMPs, activating local anti-tumor immune responses, and converting the TME from an immunosuppressive to an immunostimulatory environment $(93,103)$. However, cytokines released by MSCs recruit immune cells to the TME, further enhancing the anti-tumor immune response. Therefore, MSC carriers are considered as promising cellular vehicles for OV delivery. Assuming the high quality of MSCs and appropriate conditions of MSCs loading the virus, it is worth treating malignant tumors with such therapy, which could lead to a restrain of tumor growth progression in patients. However, further investigation is required to evaluate the effects of MSC loading viruses and explore the immune regulation mechanisms of MSCs on anti-viral and anti-tumor immune responses. 
Table I. MSCs as carriers for OV delivery.

\begin{tabular}{|c|c|c|}
\hline Author, year & Strategies & Results \\
\hline
\end{tabular}

Yoon et al, 2019,

Cancer Res

Du et al, 2017 ,

Proc Natl Acad Sci USA

Kazimirsky et al, 2016,

Stem Cell Res Ther

Kaczorowski et al, 2016, Oncotarget

Melen et al, 2016,

Cancer Lett

Leoni et al, 2015,

Oncotarget

Hoyos et al, 2015,

Mol Ther

Franco-Luzon et al, 2020,

Oncotarget

Morales-Molina et al, 2020,

Cancers (Basel)

Hammer et al, 2015,

Int J Cancer

Ong et al, 2013, J Hepatol

Castleton et al, 2014, Blood

Mader et al, 2009,

Clin Cancer Res

Hai et al, 2012,

Chin J Cancer

Ahmed et al, 2010,

Mol Ther

Hakkarainen et al, 2007 , Hum Gene Ther
MSCs loading OADs

MSCs loading OHSV

MSCs loading NDV

MSCs loading E1B19K deleted or TRAIL inserted OADs

MSCs loading genetically modified OADs

MSCs loading OHSV

MSCs loading ICOVIR 15 and Inducible Caspase 9 suicide gene (iC9) inserted OADs

MSCs loading ICOVIR5

MSCs loading E1B19K

deleted or TRAIL inserted OADs

MSCs loading MV

MSCs loading genetically modified OADs

MSCs loading OADs

MSCs loading infectious enhanced OADs
MSCs cells locate to the tumor site and lead to the accumulation of high virion levels in the tumor tissue, which eventually led to the inhibition of tumor growth.

Combination of MSCs-OHSV and an anti-PD-L1 immune checkpoint inhibitor increases the number of $\mathrm{CD}^{+}$tumor infiltrating $\mathrm{T}$ lymphocytes and significantly prolonges mice survival.

Factors secreted by MSCs infected with virus make glioma cells sensitive to the cytotoxicity. of NDV TRAIL and NDV have synergistic effect in inducing glioma cell death.

After treatment, the tumor volume decreased significantly, Ki67 and CD24 expression is decreased and caspase- 3 activity is increased.

Clinical trials confirm the safety of MSCs loading genetically modified OADs.

MSCs-OHSV significantly inhibit the brain metastasis of breast cancer in NSG mice.

MSCs loading ICOVIR15 increase the control of tumor growth and prolonge the survival of tumor-bearing mice.

MSCs carrying ICOVIR5 enhance anti-tumor. effects

This strategy increases the release of the OADs from MSCs, while MSC migration ability is not affected.

In the presence of high titer anti-measles virus antibodies, measles virus-infected MSCs can significantly induce heterocellular formation when compared with naked virus alone. In addition, MSCs accurately deliver measles virus to tumor lesions and prolong mice survival.

MSCs carrying replicable adenovirus can significantly inhibit tumor growth in vivo.

MSCs carrying OADs enhance the spread and persistence of OADs

Intravenously transplanted MSCs are mainly located in the lung, and the virus is released to advanced orthotopic breast and lung tumors to improve the efficacy.

OADs, Oncolytic Adenovirus; OHSV, Oncolytic Herpes Simplex Virus; NDV, Newcastle Disease Virus; MV, Measles Virus; MSCs, mesenchymal stem cells; MSCs-OHSV, mesenchymal stem cells loading Oncolytic Herpes Simplex Virus.

In TME, cancer-associated fibroblasts, adipocytes, Tregs, mesenchymal stromal cells and tumor-associated macrophages release numerous cytokines, such as IL-10, which support immune evasion and tumor growth (108). In recent years, oncolytic virotherapy (OVT) has been demonstrated to relieve the tumor immunosuppressive environments, and enhance anti-tumor 
immune responses $(109,110)$. OVs stimulate anti-tumor immune responses which in turn, enhance the efficacy of immune checkpoint inhibitors (ICIs) (111). For this reason, emerging evidence from preclinical and clinical trials has indicated that combined OVT and ICIs could improve the anti-tumor therapeutic efficacy (112-114). In view of the contribution of MSCs to the activation of immune responses in virotherapy, combined MSC loading OVs with ICIs could be considered as a major therapeutic area for future anti-tumor research.

\section{Acknowledgements}

Not applicable.

\section{Funding}

This study was supported by the National Natural Science Foundation of China (grant no. 81871313), the Graduate Student Innovation Program in Guizhou Province [grant no. Qian Jiao He YJSCXJH (2020) 143], Key projects of Guizhou Provincial Department of Science and Technology [grant no. Qian Ke He Zhi Cheng (2020) 4Y192], the Guizhou Provincial Natural Science Foundation [grant no. (2019)5663], the Program for Top Scientifc and Technological Talents in Guizhou Province [grant no. KY (2018)049], the Guizhou Province Science and Technology Talent Platform Project [grant no. (2019)5406], the Non-profit Central Research Institute Fund of Chinese Academy of Medical Sciences (grant nos. 2018PT31048 and 2019PT310013) and the Special Grant for Central Government Supporting Local Science and Technology Development, Science and Technology Department of Guizhou Province [grant no. (2019)4008].

\section{Availability of data and materials}

Not applicable.

\section{Authors' contributions}

$\mathrm{XW}$ and $\mathrm{ZH}$ conceived the review. $\mathrm{XW}$ wrote the review. $\mathrm{ZH}$ and $\mathrm{XZ}$ revised the review. $\mathrm{XW}, \mathrm{XZ}$ and $\mathrm{ZH}$ proofread the manuscript and revised the manuscript for intellectual content. All authors read and approved the final version.

\section{Ethics approval and consent to participate}

Not applicable.

\section{Patient consent for publication}

Not applicable.

\section{Competing interests}

The authors declare that they have no competing interests.

\section{References}

1. Hayes C: Cellular immunotherapies for cancer. Ir J Med Sci: Jul 1, 2020 (Epub ahead of print). doi: 10.1007/s11845-020-02264-w.
2. Alard E, Butnariu AB, Grillo M, Kirkham C, Zinovkin DA, Newnham L, Macciochi J and Pranjol MZI: Advances in anti-cancer immunotherapy: Car-T cell, checkpoint inhibitors, dendritic cell vaccines, and oncolytic viruses, and emerging cellular and molecular targets. Cancers (Basel) 12: 1826, 2020.

3. Hemminki O, Dos Santos JM and Hemminki A: Oncolytic viruses for cancer immunotherapy. J Hematol Oncol 13: 84, 2020.

4. Romero D: Immunotherapy: Oncolytic viruses prime antitumour immunity. Nat Rev Clin Oncol 15: 135, 2018.

5. Engeland CE and Bell JC: Introduction to oncolytic virotherapy. Methods Mol Biol 2058: 1-6, 2020.

6. Kolb EA, Sampson V, Stabley D, Walter A, Sol-Church K, Cripe T, Hingorani P, Ahern $\mathrm{CH}$, Weigel BJ, Zwiebel J and Blaney SM: A phase I trial and viral clearance study of reovirus (Reolysin) in children with relapsed or refractory extra-cranial solid tumors: A children's oncology group phase I consortium report. Pediatr Blood Cancer 62: 751-758, 2015.

7. Hamid O, Ismail R and Puzanov I: Intratumoral immunotherapy-update 2019. Oncologist 25: e423-e438, 2020.

8. Roy DG, Bell JC and Bourgeois-Daigneault MC: Magnetic targeting of oncolytic VSV-based therapies improves infection of tumor cells in the presence of virus-specific neutralizing antibodies in vitro. Biochem Biophys Res Commun 526: 641-646, 2020.

9. Schirrmacher V, van Gool S and Stuecker W: Breaking therapy resistance: An update on oncolytic newcastle disease virus for improvements of cancer therapy. Biomedicines 7: 66, 2019.

10. Mahasa KJ, de Pillis L, Ouifki R, Eladdadi A, Maini P, Yoon AR and Yun CO: Mesenchymal stem cells used as carrier cells of oncolytic adenovirus results in enhanced oncolytic virotherapy. Sci Rep 10: 425, 2020.

11. Hadrys A, Sochanik A, McFadden G and Jazowiecka-Rakus J: Mesenchymal stem cells as carriers for systemic delivery of oncolytic viruses. Eur J Pharmacol 874: 172991, 2020.

12. Naseri Z, Oskuee RK, Forouzandeh-Moghadam M and Jaafari MR: Delivery of LNA-antimiR-142-3p by mesenchymal stem cells-derived exosomes to breast cancer stem cells reduces tumorigenicity. Stem Cell Rev Rep 16: 541-556, 2020.

13. Altaner $\mathrm{C}$ and Altanerova U: Mesenchymal stem cell exosome-mediated prodrug gene therapy for cancer. Methods Mol Biol 1895: 75-85, 2019.

14. Kostadinova M and Mourdjeva M: Potential of mesenchymal stem cells in anti-cancer therapies. Curr Stem Cell Res Ther 15: 482-491, 2020.

15. Spaggiari GM, Capobianco A, Abdelrazik H, Becchetti F, Mingari MC and Moretta L: Mesenchymal stem cells inhibit natural killer-cell proliferation, cytotoxicity, and cytokine production: Role of indoleamine 2,3-dioxygenase and prostaglandin E2. Blood 111: 1327-1333, 2008.

16. Jiang XX, Zhang Y, Liu B, Zhang SX, Wu Y, Yu XD and Mao N: Human mesenchymal stem cells inhibit differentiation and function of monocyte-derived dendritic cells. Blood 105: 4120-4126, 2005.

17. Lei J, Jacobus EJ, Taverner WK, Fisher KD, Hemmi S, West K, Slater L, Lilley F, Brown A, Champion B, et al: Expression of human CD46 and trans-complementation by murine adenovirus 1 fails to allow productive infection by a group B oncolytic adenovirus in murine cancer cells. J Immunother Cancer 6: 55, 2018.

18. Koehler M, Aravamudhan P, Guzman-Cardozo C, Dumitru AC, Yang J, Gargiulo S, Soumillion P, Dermody TS and Alsteens D: Glycan-mediated enhancement of reovirus receptor binding. Nat Commun 10: 4460, 2019

19. Phillips MB, Stuart JD, Rodriguez Stewart RM, Berry JT, Mainou BA and Boehme KW: Current understanding of reovirus oncolysis mechanisms. Oncolytic Virother 7: 53-63, 2018.

20. Sakurai F, Inoue S, Kaminade T, Hotani T, Katayama Y, Hosoyamada E, Terasawa Y, Tachibana $\mathrm{M}$ and Mizuguchi $\mathrm{H}$ : Cationic liposome-mediated delivery of reovirus enhances the tumor cell-killing efficiencies of reovirus in reovirus-resistant tumor cells. Int J Pharm 524: 238-247, 2017.

21. Mahalingam D, Goel S, Aparo S, Patel Arora S, Noronha N, Tran H, Chakrabarty R, Selvaggi G, Gutierrez A, Coffey M, et al: A phase II study of pelareorep (REOLYSIN ${ }^{(R)}$ ) in combination with gemcitabine for patients with advanced pancreatic adenocarcinoma. Cancers (Basel) 10: 160, 2018.

22. Jonker DJ, Tang PA, Kennecke H, Welch SA, Cripps MC, Asmis T, Chalchal H, Tomiak A, Lim H, Ko YJ, et al: A randomized phase II study of FOLFOX6/bevacizumab with or without pelareorep in patients with metastatic colorectal cancer: IND.210, a canadian cancer trials group trial. Clin Colorectal Cancer 17: 231-239 e7, 2018. 
23. Davola ME and Mossman KL: Oncolytic viruses: How 'lytic' must they be for therapeutic efficacy? Oncoimmunology 8: e1581528, 2019.

24. Luo Y, Lin C, Zou Y, Ju F, Ren W, Lin Y, Wang Y, Huang X, Liu $\mathrm{H}, \mathrm{Yu} \mathrm{Z}$, et al: Tumor-targeting oncolytic virus elicits potent immunotherapeutic vaccine responses to tumor antigens. Oncoimmunology 9: 1726168, 2020.

25. Pidelaserra-Marti G and Engeland CE: Mechanisms of measles virus oncolytic immunotherapy. Cytokine Growth Factor Rev 56: 28-38, 2020

26. Pol JG, Bridle BW and Lichty BD: Detection of tumor antigen-specific T-cell responses after oncolytic vaccination. Methods Mol Biol 2058: 191-211, 2020.

27. Keshavarz M, Solaymani-Mohammadi F, Miri SM and Ghaemi A: Oncolytic paramyxoviruses-induced autophagy; a prudent weapon for cancer therapy. J Biomed Sci 26: 48, 2019.

28. Bommareddy PK,Zloza A, Rabkin SD and Kaufman HL: Oncolytic virus immunotherapy induces immunogenic cell death and overcomes STING deficiency in melanoma. Oncoimmunology 8: $1591875,2019$.

29. Ma J, Ramachandran M, Jin C, Quijano-Rubio C, Martikainen M, $\mathrm{Yu}$ D and Essand M: Characterization of virus-mediated immunogenic cancer cell death and the consequences for oncolytic virus-based immunotherapy of cancer. Cell Death Dis 11: 48, 2020.

30. Wang X, Shao X, Gu L, Jiang K, Wang S, Chen J, Fang J, Guo X, Yuan M, Shi J, et al: Targeting STAT3 enhances NDV-induced immunogenic cell death in prostate cancer cells. J Cell Mol Med 24: 4286-4297, 2020.

31. Shao X, Wang X, Guo X, Jiang K, Ye T, Chen J, Fang J, Gu L, Wang S, Zhang G, et al: STAT3 contributes to oncolytic newcastle disease virus-induced immunogenic cell death in melanoma cells. Front Oncol 9: 436, 2019.

32. Xu Q, Rangaswamy US, Wang W, Robbins SH, Harper J, Jin H and Cheng X: Evaluation of newcastle disease virus mediated dendritic cell activation and cross-priming tumor-specific immune responses ex vivo. Int J Cancer 146: 531-541, 2020.

33. Garg AD and Agostinis P: Cell death and immunity in cancer: From danger signals to mimicry of pathogen defense responses Immunol Rev 280: 126-148, 2017.

34. Jiang $\mathrm{H}$ and Fueyo J: Healing after death: Antitumor immunity induced by oncolytic adenoviral therapy. Oncoimmunology 3 e947872, 2014

35. Kepp O, Senovilla L, Vitale I, Vacchelli E, Adjemian S, Agostinis P, Apetoh L, Aranda F, Barnaba V, Bloy N, et al: Consensus guidelines for the detection of immunogenic cell death. Oncoimmunology 3: e955691, 2014.

36. Garg AD, Galluzzi L, Apetoh L, Baert T, Birge RB, Bravo-San Pedro JM, Breckpot K, Brough D, Chaurio R, Cirone M, et al: Molecular and translational classifications of DAMPs in immunogenic cell death. Front Immunol 6: 588, 2015.

37. Das K, Urbiola C, Spiesschaert B, Mueller P and Wollmann G: Analysis of immunological treatment effects of virotherapy in tumor tissue. Methods Mol Biol 2058: 155-177, 2020.

38. Reale A, Vitiello A, Conciatori V, Parolin C, Calistri A and Palu G: Perspectives on immunotherapy via oncolytic viruses. Infect Agent Cancer 14: 5, 2019

39. Sobol PT, Boudreau JE, Stephenson K, Wan Y, Lichty BD and Mossman KL: Adaptive antiviral immunity is a determinant of the therapeutic success of oncolytic virotherapy. Mol Ther 19: 335-344, 2011.

40. Gujar S, Pol JG, Kim Y, Lee PW and Kroemer G: Antitumor benefits of antiviral immunity: An underappreciated aspect of oncolytic virotherapies. Trends Immunol 39: 209-221, 2018.

41. Ledford H: Cancer-fighting viruses win approval. Nature 526: 622-623, 2015

42. O'Donoghue C, Doepker MP and Zager JS: Talimogene laherparepvec: Overview, combination therapy and current practices. Melanoma Manag 3: 267-272, 2016.

43. Sunshine JC, Sosman J, Shetty A and Choi JN: Successful treatment of in-transit metastatic melanoma in a renal transplant patient with combination T-VEC/Imiquimod immunotherapy. J Immunother 43: 149-152, 2020.

44. Masoud SJ, Hu JB, Beasley GM, Stewart JH IV and Mosca PJ: Efficacy of talimogene laherparepvec (T-VEC) therapy in patients with in-transit melanoma metastasis decreases with increasing lesion size. Ann Surg Oncol 26: 4633-4641, 2019.

45. Howard $\mathrm{F}$ and Muthana M: Designer nanocarriers for navigating the systemic delivery of oncolytic viruses. Nanomedicine (Lond) 15: 93-110, 2020
46. Phan M, Watson MF, Alain T and Diallo JS: Oncolytic viruses on drugs: Achieving higher therapeutic efficacy. ACS Infect Dis 4: 1448-1467, 2018

47. Rosewell Shaw A and Suzuki M: Oncolytic viruses partner with T-cell therapy for solid tumor treatment. Front Immunol 9: 2103, 2018.

48. Hwang CC, Igase M, Sakurai M, Haraguchi T, Tani K, Itamoto K, Shimokawa T, Nakaichi M,Nemoto Y,Noguchi S, et al: Oncolytic reovirus therapy: Pilot study in dogs with spontaneously occurring tumours. Vet Comp Oncol 16: 229-238, 2018.

49. Mok DZL and Chan KR: The effects of pre-existing antibodies on live-attenuated viral vaccines. Viruses 12: 520, 2020

50. Harrington K, Freeman DJ, Kelly B, Harper J and Soria JC: Optimizing oncolytic virotherapy in cancer treatment. Nat Rev Drug Discov 18: 689-706, 2019.

51. Naji A, Eitoku M, Favier B, Deschaseaux F, Rouas-Freiss N and Suganuma N: Biological functions of mesenchymal stem cells and clinical implications. Cell Mol Life Sci 76: 3323-3348, 2019.

52. Volarevic V, Markovic BS, Gazdic M, Volarevic A, Jovicic N, Arsenijevic N, Armstrong L, Djonov V,Lako M and Stojkovic M: Ethical and safety issues of stem cell-based therapy. Int J Med Sci 15: 36-45, 2018

53. Dominici M, Le Blanc K, Mueller I, Slaper-Cortenbach I, Marini F, Krause D, Deans R, Keating A, Prockop DJ and Horwitz E: Minimal criteria for defining multipotent mesenchymal stromal cells. The international society for cellular therapy position statement. Cytotherapy 8: 315-317, 2006.

54. Salmasi Z, Hashemi M, Mahdipour E, Nourani H, Abnous K and Ramezani M: Mesenchymal stem cells engineered by modified polyethylenimine polymer for targeted cancer gene therapy, in vitro and in vivo. Biotechnol Prog 36: e3025, 2020.

55. Yoon AR, Hong J, Li Y, Shin HC, Lee H, Kim HS and Yun CO: Mesenchymal stem cell-mediated delivery of an oncolytic adenovirus enhances antitumor efficacy in hepatocellular carcinoma. Cancer Res 79: 4503-4514, 2019.

56. Vangala G, Imhoff FM, Squires CML, Cridge AG and Baird SK: Mesenchymal stem cell homing towards cancer cells is increased by enzyme activity of cathepsin D. Exp Cell Res 383: 111494, 2019.

57. Kwon S, Yoo KH, Sym SJ and Khang D: Mesenchymal stem cell therapy assisted by nanotechnology: A possible combinational treatment for brain tumor and central nerve regeneration. Int J Nanomedicine 14: 5925-5942, 2019.

58. Thomas JG, Parker Kerrigan BC, Hossain A, Gumin J, Shinojima N, Nwajei F, Ezhilarasan R, Love P, Sulman EP and Lang FF: Ionizing radiation augments glioma tropism of mesenchymal stem cells. J Neurosurg 128: 287-295, 2018.

59. Choi SA, Lee JY, Kwon SE, Wang KC, Phi JH, Choi JW, Jin X, Lim JY, Kim H and Kim SK: Human adipose tissue-derived mesenchymal stem cells target brain tumor-initiating cells. PLoS One 10: e0129292, 2015.

60. Verdelli C, Vaira V and Corbetta S: Parathyroid tumor microenvironment. Adv Exp Med Biol 1226: 37-50, 2020.

61. Karagiannis K, Proklou A,Tsitoura E,Lasithiotaki I,Kalpadaki C, Moraitaki D, Sperelakis I, Kontakis G, Antoniou KM and Tzanakis N: Impaired mRNA expression of the migration related chemokine receptor CXCR4 in mesenchymal stem cells of COPD patients. Int J Inflam 2017: 6089425, 2017.

62. Armakolas A, Dimakakos A, Loukogiannaki C, Armakolas N, Antonopoulos A, Florou C, Tsioli P, Papageorgiou E, Alexandrou TP, Stathaki M, et al: IL-6 is associated to IGF-1Ec upregulation and Ec peptide secretion, from prostate tumors. Mol Med 24: 6, 2018.

63. Lejmi E, Perriraz N, Clement S, Morel P, Baertschiger R, Christofilopoulos P, Meier R, Bosco D, Buhler LH and Gonelle-Gispert C: Inflammatory chemokines MIP-1 $\delta$ and MIP-3 $\alpha$ are involved in the migration of multipotent mesenchymal stromal cells induced by hepatoma cells. Stem Cells Dev 24: 1223-1235, 2015

64. Pavon LF, Sibov TT, de Souza AV, da Cruz EF, Malheiros SM, Cabral FR, de Souza JG, Boufleur P, de Oliveira DM, de Toledo SR, et al: Tropism of mesenchymal stem cell toward $\mathrm{CD}_{133^{+}}$stem cell of glioblastoma in vitro and promote tumor proliferation in vivo. Stem Cell Res Ther 9: 310, 2018.

65. Ramirez M, Garcia-Castro J, Melen GJ, Gonzalez-Murillo A and Franco-Luzon L: Patient-derived mesenchymal stem cells as delivery vehicles for oncolytic virotherapy: Novel state-of-the-art technology. Oncolytic Virother 4: 149-155, 2015.

66. Kaczorowski A, Hammer K, Liu L, Villhauer S, Nwaeburu C, Fan P, Zhao Z, Gladkich J, Gross W, Nettelbeck DM and Herr I: Delivery of improved oncolytic adenoviruses by mesenchymal stromal cells for elimination of tumorigenic pancreatic cancer cells. Oncotarget 7: 9046-9059, 2016. 
67. Mehler VJ, Burns C and Moore ML: Concise review: Exploring immunomodulatory features of mesenchymal stromal cells in humanized mouse models. Stem Cells 37: 298-305, 2019.

68. Gao F, Chiu SM, Motan DA, Zhang Z, Chen L, Ji HL, Tse HF, Fu QL and Lian Q: Mesenchymal stem cells and immunomodulation: Current status and future prospects. Cell Death Dis 7: e2062, 2016.

69. Abbasi-Kangevari M, Ghamari SH, Safaeinejad F, Bahrami S and Niknejad H: Potential therapeutic features of human amniotic mesenchymal stem cells in multiple sclerosis: Immunomodulation, inflammation suppression, angiogenesis promotion, oxidative stress inhibition, neurogenesis induction, MMPs regulation, and remyelination stimulation. Front Immunol 10: 238, 2019.

70. Ma ZJ, Wang YH, Li ZG, Wang Y, Li BY, Kang HY and Wu XY: Immunosuppressive effect of exosomes from mesenchymal stromal cells in defined medium on experimental colitis. Int J Stem Cells 12: 440-448, 2019.

71. Carreras-Planella L, Monguio-Tortajada M, Borras FE and Franquesa M: Immunomodulatory effect of MSC on B cells is independent of secreted extracellular vesicles. Front Immunol 10: 1288, 2019.

72. Wilson A, Chee M, Butler P and Boyd AS: Isolation and characterisation of human adipose-derived stem cells. Methods Mol Biol 1899: 3-13, 2019.

73. Zhang F, Wang C, Wen X, Chen Y, Mao R, Cui D, Li L, Liu J, Chen Y, Cheng J and Lu Y: Mesenchymal stem cells alleviate rat diabetic nephropathy by suppressing $\mathrm{CD} 103^{+} \mathrm{DCs}$-mediated $\mathrm{CD}^{+} \mathrm{T}$ cell responses. J Cell Mol Med 24: 5817-5831, 2020.

74. Haddad R and Saldanha-Araujo F: Mechanisms of T-cell immunosuppression by mesenchymal stromal cells: What do we know so far? Biomed Res Int 2014: 216806, 2014.

75. Rozenberg A, Rezk A, Boivin MN, Darlington PJ, Nyirenda M, Li R, Jalili F, Winer R, Artsy EA, Uccelli A, et al: Human mesenchymal stem cells impact Th17 and Th1 responses through a prostaglandin E2 and myeloid-dependent mechanism. Stem Cells Transl Med 5: 1506-1514, 2016.

76. Khare D, Or R, Resnick I, Barkatz C, Almogi-Hazan O and Avni B: Mesenchymal stromal cell-derived exosomes affect mRNA expression and function of B-lymphocytes. Front Immunol 9: 3053, 2018.

77. Corcione A, Benvenuto F, Ferretti E, Giunti D, Cappiello V, Cazzanti F, Risso M, Gualandi F, Mancardi GL, Pistoia V and Uccelli A: Human mesenchymal stem cells modulate B-cell functions. Blood 107: 367-372, 2006.

78. Rezaei Kahmini F, Shahgaldi S and Moazzeni SM: Mesenchymal stem cells alter the frequency and cytokine profile of natural killer cells in abortion-prone mice. J Cell Physiol 235 7214-7223, 2020.

79. Xu LL, Fu HX, Zhang JM, Feng FE, Wang QM, Zhu XL Xue J, Wang CC, Chen Q, Liu X, et al: Impaired function of bone marrow mesenchymal stem cells from immune thrombocytopenia patients in inducing regulatory dendritic cell differentiation through the Notch-1/Jagged-1 signaling pathway. Stem Cells Dev 26: 1648-1661, 2017.

80. Liu Q, Zheng H, Chen X, Peng Y, Huang W, Li X, Li G, Xia W, Sun Q and Xiang AP: Human mesenchymal stromal cells enhance the immunomodulatory function of CD8(+)CD28(-) regulatory T cells. Cell Mol Immunol 12: 708-718, 2015.

81. El Omar R, Xiong Y, Dostert G, Louis H, Gentils M, Menu P, Stoltz JF, Velot E and Decot V: Immunomodulation of endothelial differentiated mesenchymal stromal cells: Impact on T and NK cells. Immunol Cell Biol 94: 342-356, 2016.

82. Cho KA, Lee JK, Kim YH, Park M, Woo SY and Ryu KH Mesenchymal stem cells ameliorate B-cell-mediated immune responses and increase IL-10-expressing regulatory B cells in an EBI3-dependent manner. Cell Mol Immunol 14: 895-908, 2017

83. Liu X, Qu X, Chen Y, Liao L, Cheng K, Shao C, Zenke M, Keating A and Zhao RC: Mesenchymal stem/stromal cells induce the generation of novel IL-10-dependent regulatory dendritic cells by SOCS3 activation. J Immunol 189: 1182-1192, 2012.

84. Ahmed AU, Rolle CE, Tyler MA, Han Y, Sengupta S, Wainwright DA, Balyasnikova IV, Ulasov IV and Lesniak MS Bone marrow mesenchymal stem cells loaded with an oncolytic adenovirus suppress the anti-adenoviral immune response in the cotton rat model. Mol Ther 18: 1846-1856, 2010.

85. Atiya H, Frisbie L, Pressimone C and Coffman L: Mesenchymal stem cells in the tumor microenvironment. Adv Exp Med Biol 1234: 31-42, 2020.
86. Cai C, Hou L, Zhang J, Zhao D, Wang Z, Hu H, He J, Guan W and Ma Y: The inhibitory effect of mesenchymal stem cells with rAd-NK4 on liver cancer. Appl Biochem Biotechnol 183: 444-459, 2017.

87. Fathi E, Sanaat $Z$ and Farahzadi R: Mesenchymal stem cells in acute myeloid leukemia: A focus on mechanisms involved and therapeutic concepts. Blood Res 54: 165-174, 2019.

88. El-Khadragy MF, Nabil HM, Hassan BN, Tohamy AA, Waaer HF, Yehia HM, Alharbi AM and Moneim AEA: Bone marrow cell therapy on 1,2-Dimethylhydrazine (DMH)-induced colon cancer in rats. Cell Physiol Biochem 45: 1072-1083, 2018.

89. Morales-Molina A, Gambera S, Cejalvo T, Moreno R, Rodriguez-Milla MA, Perise-Barrios AJ and Garcia-Castro J: Antitumor virotherapy using syngeneic or allogeneic mesenchymal stem cell carriers induces systemic immune response and intratumoral leukocyte infiltration in mice. Cancer Immunol Immunother 67: 1589-1602, 2018.

90. Ruano D, Lopez-Martin JA, Moreno L, Lassaletta A, Bautista F, Andion M, Hernandez C, Gonzalez-Murillo A, Melen G, Alemany R, et al: First-in-human, first-in-child trial of autologous MSCs carrying the oncolytic virus Icovir-5 in patients with advanced tumors. Mol Ther 28: 1033-1042, 2020.

91. RinconE,CejalvoT,KanojiaD,Alfranca A, Rodriguez-MillaMA Gil Hoyos RA, Han Y, Zhang L, Alemany R, Lesniak MS and García-Castro J: Mesenchymal stem cell carriers enhance antitumor efficacy of oncolytic adenoviruses in an immunocompetent mouse model. Oncotarget 8: 45415-45431, 2017.

92. Banijamali RS, Soleimanjahi H, Soudi S, Karimi H, Abdoli A, Seyed Khorrami SM and Zandi K: Kinetics of oncolytic reovirus T3D replication and growth pattern in mesenchymal stem cells. Cell J 22: 283-292, 2020.

93. KeshavarzM,EbrahimzadehMS,Miri SM,Dianat-MoghadamH, Ghorbanhosseini SS, Mohebbi SR, Keyvani H and Ghaemi A: Oncolytic newcastle disease virus delivered by mesenchymal stem cells-engineered system enhances the therapeutic effects altering tumor microenvironment. Virol J 17: 64, 2020.

94. Hai C, Jin YM, Jin WB, Han ZZ, Cui MN, Piao XZ, Shen XH, Zhang SN and Sun HH: Application of mesenchymal stem cells as a vehicle to deliver replication-competent adenovirus for treating malignant glioma. Chin J Cancer 31: 233-240, 2012

95. Du W, Seah I, Bougazzoul O, Choi G, Meeth K, Bosenberg MW, Wakimoto H, Fisher D and Shah K: Stem cell-released oncolytic herpes simplex virus has therapeutic efficacy in brain metastatic melanomas. Proc Natl Acad Sci USA 114: E6157-E6165, 2017.

96. Ong HT, Federspiel MJ, Guo CM, Ooi LL, Russell SJ, Peng KW and Hui KM: Systemically delivered measles virus-infected mesenchymal stem cells can evade host immunity to inhibit liver cancer growth. J Hepatol 59: 999-1006, 2013.

97. Castleton A, Dey A, Beaton B, Patel B, Aucher A, Davis DM and Fielding AK: Human mesenchymal stromal cells deliver systemic oncolytic measles virus to treat acute lymphoblastic leukemia in the presence of humoral immunity. Blood 123: 1327-1335, 2014.

98. Hammer K, Kazcorowski A, Liu L, Behr M, Schemmer P, Herr I and Nettelbeck DM: Engineered adenoviruses combine enhanced oncolysis with improved virus production by mesenchymal stromal carrier cells. Int J Cancer 137: 978-990, 2015.

99. Kazimirsky G, Jiang W, Slavin S, Ziv-Av A and Brodie C: Mesenchymal stem cells enhance the oncolytic effect of newcastle disease virus in glioma cells and glioma stem cells via the secretion of TRAIL. Stem Cell Res Ther 7: 149, 2016.

100. Melen GJ, Franco-Luzon L, Ruano D, Gonzalez-Murillo A, Alfranca A, Casco F, Lassaletta A, Alonso M, Madero L, Alemany R, et al: Influence of carrier cells on the clinical outcome of children with neuroblastoma treated with high dose of oncolytic adenovirus delivered in mesenchymal stem cells. Cancer Lett 371: 161-170, 2016.

101. Leoni V, Gatta V, Palladini A, Nicoletti G, Ranieri D, Dall'Ora M, Grosso V, Rossi M, Alviano F, Bonsi L, et al: Systemic delivery of HER2-retargeted oncolytic-HSV by mesenchymal stromal cells protects from lung and brain metastases. Oncotarget 6: 34774-34787, 2015.

102. Hoyos V, Del Bufalo F, Yagyu S, Ando M, Dotti G, Suzuki M, Bouchier-Hayes L, Alemany R and Brenner MK: Mesenchymal stromal cells for linked delivery of oncolytic and apoptotic adenoviruses to non-small-cell lung cancers. Mol Ther 23: 1497-1506, 2015.

103. Franco-Luzon L, Gonzalez-Murillo A, Alcantara-Sanchez C, Garcia-Garcia L, Tabasi M, Huertas AL, Chesler L and Ramirez M: Systemic oncolytic adenovirus delivered in mesenchymal carrier cells modulate tumor infiltrating immune cells and tumor microenvironment in mice with neuroblastoma. Oncotarget 11: 347-361, 2020 
104. Morales-Molina A, Rodriguez-Milla MA, Gimenez-Sanchez A, Perise-Barrios AJ and Garcia-Castro J: Cellular virotherapy increases tumor-infiltrating lymphocytes (TIL) and decreases their PD- $1^{+}$subsets in mouse immunocompetent models. Cancers (Basel) 12: 1920, 2020.

105. Mader EK, Maeyama Y, Lin Y, Butler GW, Russell HM, Galanis E, Russell SJ, Dietz AB and Peng KW: Mesenchymal stem cell carriers protect oncolytic measles viruses from antibody neutralization in an orthotopic ovarian cancer therapy model. Clin Cancer Res 15: 7246-7255, 2009.

106. Hakkarainen T, Sarkioja M, Lehenkari P, Miettinen S, Ylikomi T, Suuronen R, Desmond RA, Kanerva A and Hemminki A: Human mesenchymal stem cells lack tumor tropism but enhance the antitumor activity of oncolytic adenoviruses in orthotopic lung and breast tumors. Hum Gene Ther 18: 627-641, 2007.

107. Ocansey DKW, Pei B, Yan Y, Qian H, Zhang X, Xu W and Mao F: Improved therapeutics of modified mesenchymal stem cells: An update. J Transl Med 18: 42, 2020.

108. Najafi M, Goradel NH, Farhood B, Salehi E, Solhjoo S, Toolee H, Kharazinejad E and Mortezaee K: Tumor microenvironment: Interactions and therapy. J Cell Physiol 234: 5700-5721, 2019.

109. Oh CM, Chon HJ and Kim C: Combination immunotherapy using oncolytic virus for the treatment of advanced solid tumors. Int J Mol Sci 21: 7743, 2020.

110. Sostoa J, Dutoit V and Migliorini D: Oncolytic viruses as a platform for the treatment of malignant brain tumors. Int $\mathrm{J}$ Mol Sci 21: 7449, 2020.
111. Sivanandam V, LaRocca CJ, Chen NG, Fong Y and Warner SG: Oncolytic viruses and immune checkpoint inhibition: The best of both worlds. Mol Ther Oncolytics 13: 93-106, 2019.

112. Heinio C, Havunen R, Santos J, de Lint K, Cervera-Carrascon V, Kanerva $A$ and Hemminki A: TNFa and IL2 encoding oncolytic adenovirus activates pathogen and danger-associated immunological signaling. Cells 9: 798, 2020.

113. Ribas A, Dummer R, Puzanov I, VanderWalde A, Andtbacka RHI, Michielin O, Olszanski AJ, Malvehy J, Cebon J, Fernandez E, et al: Oncolytic virotherapy promotes intratumoral T cell infiltration and improves Anti-PD-1 immunotherapy. Cell 174: 1031-1032, 2018.

114. Sun L, Funchain P, Song JM, Rayman P, Tannenbaum C, Ko J, McNamara M, Marcela Diaz-Montero C and Gastman B: Talimogene laherparepvec combined with anti-PD-1 based immunotherapy for unresectable stage III-IV melanoma: A case series. J Immunother Cancer 6: 36, 2018.

This work is licensed under a Creative Commons Attribution-NonCommercial-NoDerivatives 4.0 International (CC BY-NC-ND 4.0) License. 